\title{
Multiple conditioned analysis of the Turbulent Stratified Flame A
}

\author{
Thabo Stahler ${ }^{1}$, Dirk Geyer ${ }^{2}$, Gaetano Magnotti ${ }^{3}$, Philipp Trunk ${ }^{1}$, Matthew J. Dunn ${ }^{4}$, \\ Robert S. Barlow ${ }^{3}$, Andreas Dreizler ${ }^{1}$ \\ ${ }^{1}$ : Fachgebiet Reaktive Strömungen und Messtechnik (RSM), Technische Universität Darmstadt, Jovanka- \\ Bontschits-Str. 2, 64287 Darmstadt \\ ${ }^{2}$ : Thermodynamik und Alternative Antriebe, Hochschule Darmstadt, Schöfferstrasse 3, 64295 Darmstadt \\ 3: Sandia National Laboratories, Livermore, CA, USA \\ ${ }^{4}$ : The School of Aeronautical, Mechanical and Mechatronic Engineering, University of Sydney
}

Corresponding author:

Dirk Geyer
Email: dirk.geyer@h-da.de

$F B M K$

University of Applied Sciences Darmstadt

Schöfferstrasse 3

D-64295 Darmstadt

\section{Colloquium: Turbulent Combustion}

\section{Word Count According to Method 1}

Word equivalent lengths:

Main text (Introduction -> Acknowledgments) $=4520$ words

Equations: 2, $\quad 30$ words

Nomenclature: $\mathrm{n} / \mathrm{a} \quad 0$ words

References: 16, 315 words

Tables: $0, \quad 0$ words

Figures and Captions:

Figure 1: Height: $60 \mathrm{~mm}, 1 \mathrm{col}$, words including caption: 200

Figure 2: Height: $47 \mathrm{~mm}, 1 \mathrm{col}$, words including caption: 164

Figure 3: Height: $53 \mathrm{~mm}, 2$ col, words including caption: 290

Figure 4: Height: $47 \mathrm{~mm}, 1 \mathrm{col}$, words including caption: 167

Figure 5: $\quad$ Height: $47 \mathrm{~mm}, 1 \mathrm{col}$, words including caption: 173

Figure 6: Height: $47 \mathrm{~mm}, 1 \mathrm{col}$, words including caption: 167

Figure 7: Height: $47 \mathrm{~mm}, 1 \mathrm{col}$, words including caption: 159

Total Figures: $\quad 1321$ words

Total count: 6199

Hereby we affirm that we do not intend to use color reproductions in the printed proceedings 


\section{Abstract}

To explore the effect of stratification on lean premixed combustion in a turbulent flow, an experimental investigation on the TSFA flame of the Darmstadt stratified burner is conducted. Spatially highly resolved major species concentrations and temperature are measured by 1D Raman-Rayleigh. Temperature gradients from line data are corrected to the flame front normal by the means of Crossed Planar Rayleigh Imaging. A conditioning based on multiple criteria relevant for stratified combustion is applied to the large dataset and allows to analyze the impact of stratification on the flame structure. Conditioning criteria are the local equivalence ratio $(\phi=0.75)$, the local thermal progress variable $(c=0.54)$, the stratification level (instantaneous flame-normal gradient in equivalence ratio), and a temperature difference over the 1D probe volume, ensuring the flame front is included. Conditionally averaged quantities such as temperature profiles in the flame-normal coordinate system, equivalence ratio, $\mathrm{H}_{2}$ mass fractions, and temperature gradients are parameterized on the local equivalence ratio gradient in order to understand the impact of stratification on the flame structure. The temperature profiles in this back-supported configuration are more affected on the reactant side than on the product side by stratification. In contrast to that, equivalence ratios as well as mass fractions of hydrogen are found to be sensitive to stratification on the product side as well as in the reactants. Profiles are altered even in the reaction zone by enhancing the equivalence ratio gradients, which is in contrast to results obtained in the Cambridge/Sandia configuration. This finding indicates the influence of turbulence on stratified combustion in the thin-reaction-zone regime, as characterized for instance by the difference in Karlowitz and Damköhler numbers for the Darmstadt versus the Cambridge/Sandia configuration.

\section{Keywords:}

Turbulent combustion, Lean-premixed combustion, Stratified flames, Multiple conditioned data analysis, Raman/Rayleigh scattering 


\section{Introduction}

Equivalence ratio stratification is a key technology for future combustors since it ensures flame stabilization within the more rich premixed flame zone as well low NOx emissions and increasing efficiencies in the lean mixtures. Consequently, a number of numerical as well as experimental studies have been recently conducted on stratified flames as summarized by Masri [1]. Quantities such as the instantaneous equivalence ratio gradient (stratification level), the length scale on which equivalence ratio variations take place, and the equivalence ratio local to the flame front are conditions relevant to stratified flames [2]. Also, the direction of the stratification has to be distinguished. Products are closer to stoichiometric than the reactants in back supported stratified flames and vice versa in front supported flames. Back support leads to a higher flux of heat and radicals into the flame front for laminar lean flames $[1,3]$. Conditions such as temperature or laminar flame speed are thereby increased relative to a corresponding premixed flame. In order to examine the influence of turbulence on flame structure, the determining factors of stratified combustion have to be isolated. Vena et al. [4] combined planar laser induced fluorescence (LIF) of $\mathrm{CH}_{2} \mathrm{O}$ and $\mathrm{OH}$-LIF to investigate the heat release rate for various mean stratification levels. Sweeney et al. [5] conditioned 1D-Raman-Rayleigh data from the Cambridge/Sandia stratified bluff-body burner based on multiple criteria: (a) the instantaneous stratification level (b) the local equivalence ratio (c) the reaction progress variable and (d) a temperature difference over a defined length. Recently, Kamal et al. [6] reported on conditioning only on the local equivalence ratio for the same configuration to investigate global features of a flame series.

The Darmstadt Turbulent Stratified Burner employed here is complementary to the Cambridge/Sandia configuration and has been widely used as a test case for model development [7]. A series of lean turbulent stratified flames (TSF) stabilized by an inner pilot flame are produced [8] which have been the subject of experimental as well as numerical work $[2,7,8]$.

The two configurations have different positions within the flame regime diagram. The TSF series is located at the low Karlowitz $(1.2 \leq \mathrm{Ka} \leq 2.1)$ end of the thin-reaction-zone regime at Damköhler numbers of $50 \leq \mathrm{Da} \leq 80$ [2], while the flame fronts of the Cambridge/Sandia flames are within the same regime 
but at lower Damköhler $(0.6 \leq \mathrm{Da} \leq 1.0)$ and higher Karlovitz $(7.6 \leq \mathrm{Ka} \leq 40)$ numbers. This work explores the effect of stratified combustion in one TSF flame in detail by using a method of multiple conditioning similar to that in [5] to isolate the effects of stratification. Thereby, the impact of stratification on the flame structure, with respect to its position in the regime diagram, is analyzed.

\section{Experimental Setup}

\subsection{Darmstadt Stratified Burner}

Only a brief description of the Darmstadt stratified burner will be given here; more detailed information is available in $[2,8]$. As illustrated by the vertical lines in Fig. 1, the burner consists of three concentric tubes with inner diameters of $14.8,37$ and $60 \mathrm{~mm}$. The tubes are staged to enable optical access for the measurements, and the two annular gaps are referred to as slot $1\left(S_{1}\right)$ and slot $2\left(S_{2}\right)$, respectively. A flame holder, which is located inside the central tube $40 \mathrm{~mm}$ upstream of the nozzle exit, is used to stabilize a pilot flame (P) at an equivalence ratio of $\Phi=0.9$. Thereby, a fully reacted flow with uniform mass fraction profiles at the burner exit is provided. The velocity of the reacted pilot flow matches the exit velocity of the unreacted flow from $S_{1}$.

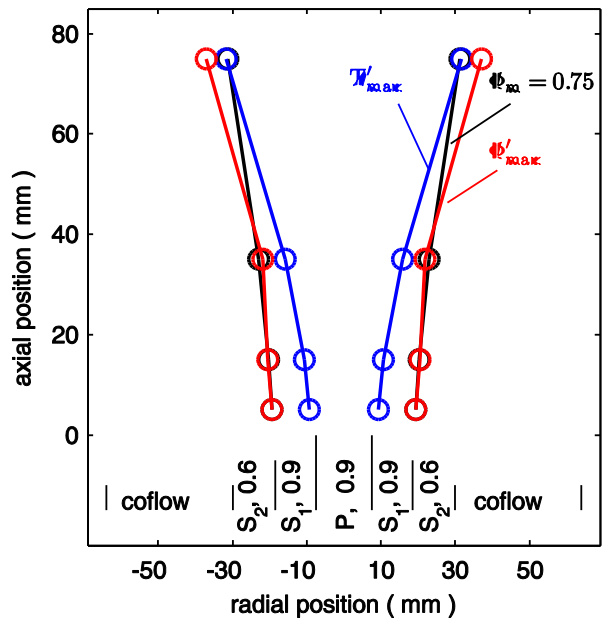

Fig. 1: Illustration of the burner configuration and mean radial position of the center of the mixing layer $\phi_{m}=$ $\frac{\phi_{1}+\phi_{2}}{2}$ and the center of the flame brush at $T_{\max }^{\prime}$. Location of the maximum in scalar mixing is marked by $\phi_{\max }^{\prime}$. Numbers at burner exit are respective equivalence ratios in $S_{1}, S_{2}$ and $P$.

The turbulent stratified flame TSFA [8] is stabilized on the burner at equivalence ratios of $\phi=0.9$ and $\phi=0.6$ for $S_{1}$ and $S_{2}$ respectively. Bulk velocities are $10 \mathrm{~m} / \mathrm{s}$ for $S_{1}$ and $S_{2}$, with the intention of 
investigating fuel stratification between $S_{1}$ and $S_{2}$ without mean shear across the turbulent mixing layer. The burner is placed inside an air coflow (inner diameter $480 \mathrm{~mm}, u_{\text {coflow }}=0.1 \mathrm{~m} / \mathrm{s}$ ) to create defined boundary conditions. Information about the flow field characteristics can be found in $[2,8]$. The profiles illustrated in Fig. 1 will be discussed in section 3.

\subsection{Measurement Techniques}

Multi-scalar line measurements of temperature (Rayleigh scattering) and major species (Raman scattering and CO-LIF) were performed at the Turbulent Combustion Laboratory at Sandia. The setup has been described in $[9,10]$. 1D Raman-Rayleigh scattering was combined with two photon CO-LIF to instantaneously measure species concentration $\left(\mathrm{N}_{2}, \mathrm{O}_{2}, \mathrm{CH}_{4}, \mathrm{H} 2 \mathrm{O}, \mathrm{CO}, \mathrm{CO}_{2}, \mathrm{H}_{2}\right)$ and temperature along a 6-mm segment of the combined laser beams. Four frequency doubled Nd:YAG lasers with combined $1.4 \mathrm{~J} /$ pulse and a beam diameter $\left(1 / e^{2}\right)$ of $220 \mu \mathrm{m}$ were used for Raman and Rayleigh excitation. For two photon CO-LIF a UV laser at $230.1 \mathrm{~nm}$ wavelength, overlapping the Nd:YAG laser beams, was employed.

The scattered light was collected using an achromatic lens system and focused into a custom detection system. Non-intensified, low noise, CCD cameras were used for Raman and Rayleigh signal detection. Gating for the Raman and Rayleigh cameras ( $3.9 \mu \mathrm{s}$ (FWHM) and $300 \mu \mathrm{s}$, respectively) was provided by custom-built rotating shutters. An intensified CCD camera was used for CO fluorescence signal detection.

The instantaneous 3D flame orientation was determined using crossed-planar Rayleigh imaging (CPRI) at $355 \mathrm{~nm}$. The two laser sheets intersected along the multi-scalar probe line and were aligned at $\pm 34^{\circ}$ with respect to the burner axis, as described in $[5,10]$. Two intensified CCD cameras recorded the 2D Rayleigh scattering image.

For results presented here, the projected pixel spacing of the Raman/Rayleigh/CO-LIF line data was 0.02 $\mathrm{mm}$ along the laser axis. The optical resolution of the line-imaging system was limited to $\sim 0.06 \mathrm{~mm}$ by the large-diameter, achromatic collection lens system. Effective spatial resolution can be further limited by the beam diameter, the angle between the flame and the beam, and blurring caused by the flame 
itself. Focusing and alignment of all cameras was accomplished by placing a multi-hole target at the object plane and back-illuminating it through a diffuser at appropriate wavelengths.

\subsection{Data Evaluation}

Raman-Rayleigh-LIF data evaluation was performed using the hybrid method described in [11]. This method is based on theoretically simulated Raman spectra, except for methane where a response function based on calibrations is used. Calibration coefficients for the different species as well as normalization curves to correct for the different throughput along the 1D probe volume were obtained by measuring several different cold flows as well as two different types of calibration flames over an equivalence ratio range from 0.7 to 1.3 . Representative values for precision and repeatability in scalar measurements are given in [10]. The precision of the temperature is $0.7 \%$ in a stoichiometric flat flame and the precision of $\mathrm{H}_{2}$ is $7.0 \%$ in slightly rich flat flame. A spatial oversampling and wavelet denoising methodology was applied to the 1D Raman-Rayleigh-LIF data to simultaneously improve SNR and enable effective spatial resolution close to the optical resolution of the system. The wavelet denoising algorithm, termed wavelet adaptive thresholding and reconstruction (WATR), has been described in [5]. Gradients along the 1D measurement volume were calculated using a fourth order implicit scheme [5].

The CPRI provides the instantaneous 3D flame orientation along the line image probe volume. Images from both planes where first spatially registered, overlapped, corrected for mean dark background, and normalized by images from homogeneous air to correct for the effects of the non-uniform beam profile and optical throughput. For contour extraction the images were first upsampled by a factor of five and segmented by applying an adaptive nonlinear diffusion algorithm. In an iterative procedure the PDF of the gradient image was monitored, and the process was aborted as soon as the steepest gradients were altered. Contours were extracted by applying the canny edge algorithm in the segmented image. Tangent vectors of the in-plane contours were estimated using analytically calculated derivatives of a spline fitted to the contour data. After transforming the tangent vectors in the inclined orientation of the crossed sheets, the instantaneous flame normal pointing to the products was determined by the cross 
product of the two tangent vectors as described in [10]. Thus, the angle $\Theta$ between the flame normal and laser line was obtained and used to correct the measured 1D projection to its 3D value.

\subsection{Multiple Conditioning}

The objective of the current investigation is to examine the impact of stratification on the flame microstructure. To understand these effects the data have at least to be conditioned on the local equivalence ratio and the local thermal progress variable. The thermal progress variable is used here because temperature measurements by Rayleigh scattering have lower noise than species measurement by Raman scattering, and this is particularly important in evaluating gradients. The local equivalence ratio was derived for each location within the 1D Raman/Rayleigh using the measured mole fractions as [3]:

$\phi=\frac{X_{\mathrm{CO}_{2}}+2 X_{\mathrm{CH}_{4}}+X_{\mathrm{CO}+0.5\left(X_{\mathrm{H}_{2} \mathrm{O}}+X_{\mathrm{H}_{2}}\right)}}{X_{\mathrm{CO}_{2}}+X_{\mathrm{O}_{2}}+0.5\left(X_{\mathrm{CO}}+X_{\mathrm{H}_{2} \mathrm{O}}\right)}$.

A thermal progress variable $c$ was calculated, based on the equilibrium temperature $T_{e q}(\phi)$ of the local equivalence ratio, the local temperature $T$ and the ambient temperature, as

$c=\left(T-T_{a m b}\right) /\left(T_{e q}(\phi)-T_{a m b}\right)$.

Different approaches to condition data from stratified flames have been employed in the literature, depending on the applied diagnostic techniques and the aims of the investigation. Robin et al. [12] conditioned on the $500 \mathrm{~K}$ isotherm in the preheat zone. Vena et al.[4] employed the flame front derived from combined tracer PLIF / $\mathrm{CH}_{2} \mathrm{O}$-LIF measurements at a progress variable of 0.5 to condition mean equivalence ratio gradients. Sweeney et al. [5] conditioned on the location of the peak CO mass fraction as a surrogate for the location of the maximum heat release in laminar lean premixed flames. However, Richardson et al. [3] pointed out that the impact of stratification on the flame is most prominent at the largest gradients of the thermal progress variable, which is equivalent to the largest temperature gradients considering the definition of $c$ used in this work. Additionally, temperature gradients offer the advantage that they can be directly quantified by the experimental system. 
Calculations of unstrained laminar flames (Cantera2.1 [13] and GRI3.0 [14], including multicomponent diffusion and Soret effect) over the range of equivalence ratios existing in the mixing layer between $\mathrm{S}_{1}$ and $S_{2}$ show that the peak of $\nabla T$ shifts from $c=0.49$ in more rich mixtures $(\phi=0.90)$ towards $c=0.63$ in lean mixtures $(\phi=0.60)$. The largest $\nabla T$ at the mean equivalence ratio $\left(\phi_{m}=0.75\right)$ of the mixing layer is located at $c=0.54$. Accordingly, selecting $c=0.54$ to condition on allows well to investigate the impact of stratification on the turbulent flame over the range of equivalence ratios prevalent in the mixing layer.

To allow consistent comparison, the local equivalence ratio within simulated laminar flames has been computed using Eq. 1. Effects of stratification are discussed in relation to unstrained and strained (1000 1/s) laminar flames. To coincide with the conditioning of their turbulent counterparts the local equivalence ratio has to be 0.75 at $c=0.54$ in the simulations, which corresponds to a global values of $\phi=0.808$ and $\phi=0.772$ for the unstrained and strained flame, respectively. The difference is an effect of differential diffusion within the flame. This approach allows for the isolation of the effect of equivalence ratio gradient directly in the reaction zone [3]. The temperature of the unstrained laminar flame at $c=0.54(1247 \mathrm{~K})$ is used later in the discussion of the temperature conditioned data to indicate the location of the maximum temperature gradient.

To ensure that the 1D Raman-Rayleigh line volume crosses the flame brush and is not tangent to it, profiles with a temperature change less than $600 \mathrm{~K}$ were not considered in the analysis. For the flame position considered in this study the flame angle derived from the CPRI has a mean of $\bar{\Theta}=35.60^{\circ}$ and a standard deviation of $\sigma_{\Theta}=12.20^{\circ}$. To minimize the effect of erroneous 3D corrections, the range of $\Theta$ is limited to $55^{\circ}$.

We computed the quasi 3D flame-normal coordinate $r^{*}$ by projecting data from the instantaneous 1D Raman/Rayleigh profiles onto the direction of the flame normal vector obtained from CPRI. The origin of $r^{*}$ corresponds to $c=0.54$. The stratification level is quantified by the 3D corrected metric $\Delta \phi / \Delta r^{*}$. For that purpose the data were confined to $\pm 0.30 \mathrm{~mm}$ around the origin of $r^{*}$, thereby using a simplified 2- 
point equivalence ratio gradient across the instantaneous flame. This 2-point metric is used because the contribution of noise to local gradients calculated at the measurement resolution is comparable to the effect of stratification. Precision (rms uncertainty) in $\Delta \phi / \Delta r^{*}$ is roughly $0.02 \mathrm{~mm}^{-1}$, based on uncertainty in $\Phi$ measured in premixed flat flames.

All conditional averages in the present work have been calculated as ensemble averages without density weighting. For much of the analysis, temperature is one of the conditioning variables, so density fluctuations within a given sample bin are minor, and density weighting would not significantly change the results or conclusions.

\section{Results and Discussion}

Figure 1 shows the radial positions of the mean mixing layer, specified to be centered at $\phi_{m}=0.75$ between the flows emanating from $\mathrm{S}_{1}\left(\phi_{1}=0.90\right)$ and $\mathrm{S}_{2}\left(\phi_{2}=0.60\right)$. The position of the mean flame brush, also shown, is defined as the location of the largest temperature fluctuations of $T_{\max }^{\prime}$ in each radial profile. At an axial position of $z=75 \mathrm{~mm}$ the mean flame brush $(r=31.7 \mathrm{~mm}$ ) has radially progressed to intersect the mean mixing layer $(r=31.3 \mathrm{~mm})$. After the location of this intersection was identified, a total of 31,000 shots were acquired from radial positions $27-33 \mathrm{~mm}$ and $30-36 \mathrm{~mm}$ for the multi-conditioned analysis. Selecting the intersection of these two layers as the location to apply the multi-conditioned analysis ensures that the impact of equivalence ratio stratification on the flame microstructure in a turbulent flow can be examined best.

\subsection{Single shot trajectories}

Typical trajectories of the methane mass fraction over the local equivalence ratio for $\left.\phi\right|_{c=0.54}=0.75 \pm$ 0.03 and a low level of stratification $\left(\Delta \phi / \Delta r^{*}=0.025 \mathrm{~mm}^{-1}\right.$, red lines), as well as a high level $\left(\Delta \phi / \Delta r^{*}=0.195 \mathrm{~mm}^{-1}\right.$, black lines) are shown in Fig. 2. Each trajectory represents a single laser shot and displays results along the 1D probe volume. The colored symbols represent the temperature at each radial position with $20 \mu \mathrm{m}$ data spacing. Maximum possible methane mass fraction is depicted by the dashed line, representing pure mixing of a non-reacting stratified flow. The high stratification trajectories 
show a significant decrease in equivalence ratio with decreasing temperature. This is typical for a turbulent stratified flame that is back supported in the mean [15], burning from richer towards leaner mixture.

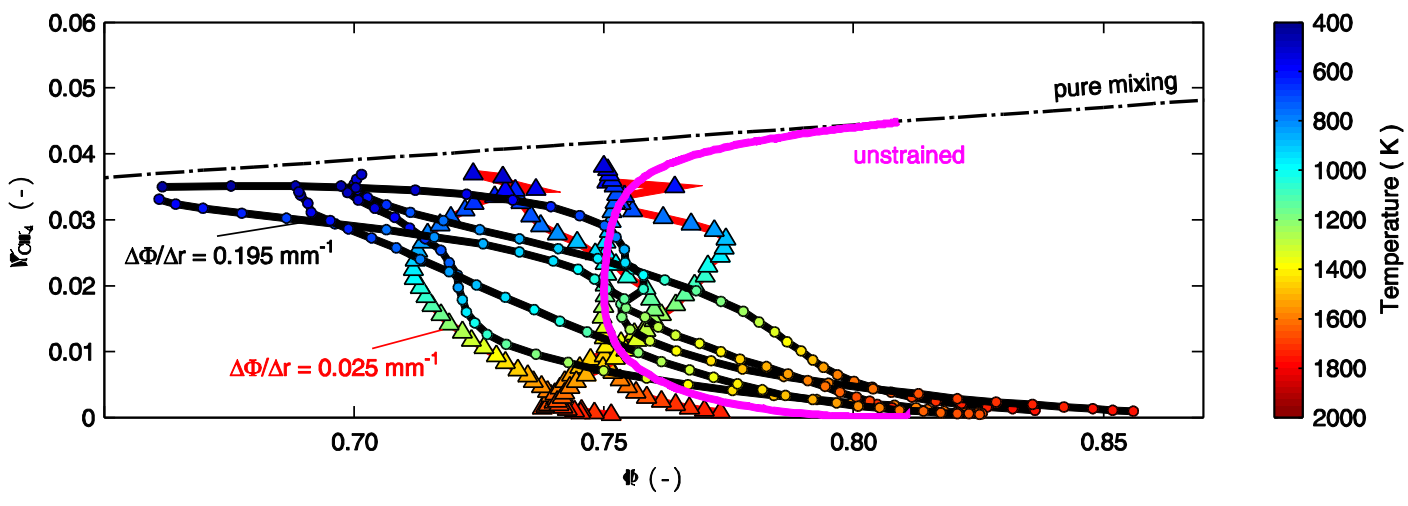

Fig. 2: $\mathrm{CH}_{4}$-trajectories for $\left.\phi\right|_{c=0.54}=0.75 \pm 0.03$ over the local equivalence ratio at two different levels of stratification. Low stratification ( $\Delta$ symbols), high stratification (o symbols), and pure mixing (black, dash-dotted). An unstrained premixed flame at matching local equivalence ratio is included for comparison.

At the highest temperatures the trend of the low stratification trajectories is similar to the laminar flame. Temperature and fuel mass fraction are not independent quantities in premixed flames: when the fuel mass fraction goes down the temperature increases. Equivalence ratios are decreasing as the temperatures decrease due to differential diffusion effects. Neither turbulence nor stratification appears to have a strong impact on the structure of the reaction zone itself at the high temperature end. In the mid temperature range, corresponding to the preheat zone, the flame structure of the low stratification trajectories is perturbed. This could be caused by turbulent eddies which are able to penetrate into the preheat zone but not into the thinner, highly viscous reaction zone. The increase in $\phi$ at low temperatures in some of the experimental profiles is similar to that of the unstrained laminar flame and is again associated with differential diffusion effects. In contrast, the high stratification trajectories show a severe impact of stratification on the flame structure. The high temperature reaction zone of the back supported flame is located at larger equivalence ratios than for the low stratification case, and the temperature decays rapidly in the direction of the smaller equivalence ratios. Perturbations of the profiles seem to be much less pronounced here except at the lowest temperatures. 


\subsection{Pdfs of stratification}

The main objective of this investigation is to examine the effect of stratification on the flame microstructure. Note that the flame is within the thin-reaction-zone regime. To achieve this, the local equivalence ratio to condition on must be close to the center of the mixing layer $\left(\phi_{m}=0.75\right)$ and provide a sufficient number of samples after applying the multiple conditioning. Beside $\phi_{m}=0.75$ local equivalence ratios of 0.65 and 0.85 , located at the lean and the rich edge of the mixing layer were considered, with sampling intervals of $\Delta \phi= \pm 0.03$ after conditioning on $c=0.54$. Probability density distributions (pdf) of stratification for all cases are shown in Fig. 3 and reveal that conditioned stratification levels tend to increase moving across the flame brush from richer to leaner conditions. Data from each equivalence ratio are grouped in three levels of stratification $-0.07 \leq \Delta \phi / \Delta r^{*} \leq 0.07$, $0.07<\Delta \phi / \Delta r^{*} \leq 0.15$ and $0.15<\Delta / \Delta r^{*} \leq 0.23 \mathrm{~mm}^{-1}$ for further analysis. However, the number of samples (143) recorded for the $\left.\phi\right|_{c=0.54}=0.65$ condition was too small for reliable statistics. The same limitation holds for $\left.\phi\right|_{c=0.54}=0.85$, particularly for larger stratifications. Therefore, only the $\left.\phi\right|_{c=0.54}=0.75$ case (545 samples) will be examined in the following.

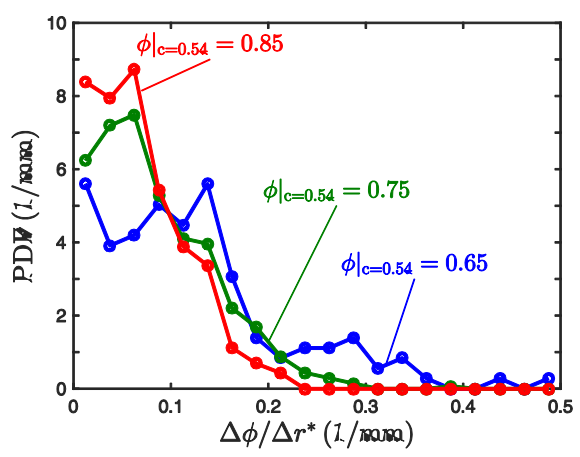

Fig. 3: Probability density distribution of stratification conditioned for three different equivalence ratios.

\subsection{Temperatures over flame-normal coordinate}

Insight into the interaction of stratified flames with turbulence is provided by examining the conditional mean of spatial temperature profiles plotted over the flame-normal coordinate $r^{*}$ in Fig. 4. All levels of stratification as well as the laminar flames shown for comparison intersect at the origin of $r^{*}$. With 
increasing stratification the temperature profiles become steeper. This reflects the increase in equivalence ratio with the stratification level for $r^{*}>0$ (products) and the decrease of $\phi$ for $r^{*}<0$ (reactants). On the product side the increase of the stratification level has a small impact; on the reactant side the impact of stratification on the conditional mean temperature is larger. These observations are discussed in the following.

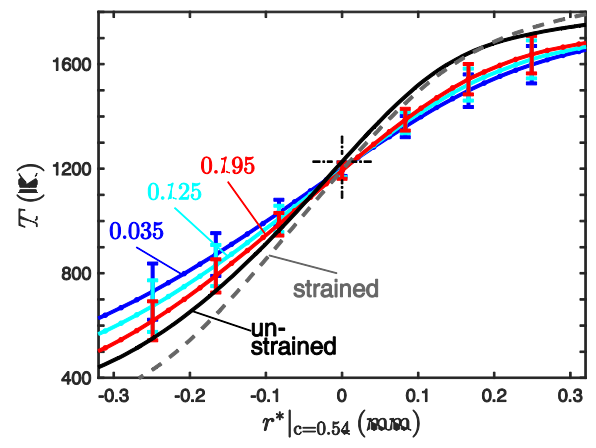

Fig. 4: Mean and standard deviation of temperature over flame-normal coordinate $r^{*}$, parameterized on three different levels of stratification in $\mathrm{mm}^{-1}$; low (blue), mid (turquoise), high (red). Unstrained and strained (1000 $\left.\mathrm{s}^{-1}\right)$ premixed laminar flame at equivalent local equivalence ratio for comparison.

Differences on the reactant side at least partly result from transport effects. Steeper temperature profiles as a consequence of stratification have been assigned to an enhanced transport of radicals and heat from the reaction zone into the preheat layer in laminar lean, back supported flames. Richardson et al. [3] revealed that laminar stratified back supported flames showed an increase in temperature on the product side and a decrease on the reactant side in comparison to premixed flames. The same holds true within the turbulent flame for the different stratification levels. As the stratification level is increased the temperatures increase on the product side while they decrease on the reactant side. This is caused by afore mentioned enhanced transport as well as by the change in the local stoichiometry. The local adiabatic flame temperature is increasing with the stratification level for the products due to larger local equivalence ratios and vice versa for the reactants. However, the increases in the mean temperatures observed for the higher stratification levels in the products are distinctly smaller than the corresponding temperature differences on the reactant side. Conditional mean temperatures in the turbulent stratified flames, even including the standard deviations, show in the mean a wider profile than in the 
corresponding laminar strained and even the laminar unstrained premixed flame. A similar widening of the temperature profiles by turbulence has been observed for a lean premixed $(\phi=0.75)$, bluff body stabilized flame in a case with inceased bulk velocities and turbulence levels by Magnotti and Barlow [10]. Moreover, Tamadonfar and Gülder [16] recently discussed the impact of turbulence and the Ka number on the thickness of the preheat zone as well as of the reaction zone in turbulent, lean premixed flames. They observed larger thicknesses of the preheat zone as well as of the reaction zone for equivalence ratios varying from 0.8 to 1.0 . This equivalence ratio range corresponds to the product side of the multi-conditioned data here and agrees with the findings in Fig. 4. However, [16] reported a decrease of the width of the preheat zone and the reaction zone for equivalence ratios in between 0.6 to 0.7 , which was not observed in the current study. Further parametric studies are required to investigate these differing findings more deeply.

Turbulence-chemistry interaction results in the products in temperatures well below the ones in laminar flames as has been observed before $[5,9,10]$. A comparison with a steady strained flame $(10001 / \mathrm{s})$ at the same local equivalence ratio at $c=0.54$ underlines that the lower conditional mean temperatures on the product side of the turbulent stratified flames are not caused by tangential strain since this effect results in laminar premixed flames in even higher temperatures profile. Effects like unsteady turbulent transport involving different components of strain, flame curvature as well as flame-flame interactions onto the stratified turbulent flame structure need to be explored in more detail to gain insight into the widening of the flame front and the related temperature profiles. The conditional mean profiles in the turbulent stratified flames are in general wider and less steep than in the corresponding laminar premixed flames.

\subsection{Temperature space}

Figure 5 shows multi-conditioned results for equivalence ratio in temperature space. The impact of stratification is clearly visible on the reactant side (low temperatures) in the mean of the equivalence ratio. The profiles are almost linear and increasingly inclined with higher stratification. At the smallest stratification level the impact of strain rather than stratification is visible by comparison to the 
unstrained and strained premixed flame. All profiles intersect close to the temperature where the unstrained premixed flame exhibits $c=0.54$. On the product side (high temperatures) above this intersection point the situation is different. The lowest stratifications show an increasing gain in equivalence ratio at the high temperature end. Such an increase is also visible in the two laminar flames and is caused by differential molecular diffusion. Note, that the maximum heat release $Q_{\max }$ in the unstrained flame occurs at a temperature of $1577 \mathrm{~K}$ and is in laminar flames slightly shifted to higher temperatures for lean stratified flames [3]. For the highest stratification level $\left(\Delta \phi / \Delta r^{*}=0.195 \mathrm{~mm}^{-1}\right)$ the mean of $\phi$ is an almost straight line on the reactants side, and the inclination is not significantly altered within the temperature range of the products. Still, differential molecular diffusion effects seem also to be superimposed on the stratification in the equivalence ratio profiles for both intermediate and highest stratification level.

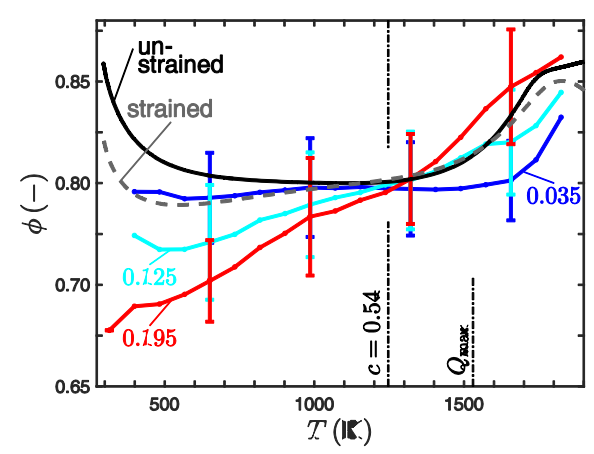

Fig. 5: Mean and standard deviation of $\phi$ in temperature space for two equivalence ratios, parameterized on three different levels of stratification (in $\left.\mathrm{mm}^{-1}\right)$. Unstrained and strained $\left(1000 \mathrm{~s}^{-1}\right)$ premixed laminar flame at equivalent local equivalence ratio for comparison. Vertical lines indicate location of maximum temperature gradient $(c=0.54)$ and location of maximum heat release in temperature space for unstrained flame.

Sweeney et al. [5] found an independence of the equivalence ratio profiles on the stratification level on the product side of a lean stratified flame employing a similar conditioning as here. They identified their flame to be within the thin-reaction-zone regime, and they recognized differential diffusion effects. The Cambridge/Sandia flame exhibits a lower Damköhler and a higher Karlovitz number (see Introduction) and thereby a larger impact of turbulence on the flame front compared to flame TSFA. Stratification has a recognizable impact on the flame microstructure in flame TSFA even in the region of the reaction zone because of the smaller impact of turbulence onto the flame structure. 
Figure 6 shows the species mass fraction of $\mathrm{H}_{2}$ over temperature for three different levels of stratification. $Y_{H_{2}}$ is larger in the products for the higher stratifications as a consequence of the gain in equivalence ratio by increased equivalence ratio gradients. The highly diffusive $\mathrm{H}_{2}$ is preferentially transported towards the preheat zone [5] on the lean side and leads to higher levels of $Y_{\mathrm{H}_{2}}$ therein. Note, that the local equivalence ratio as well as the temperature for larger stratifications is smaller in the preheat zone (Fig. 5). The first effect increases the diffusion of light species while the second slows it down in respect to the other major species.

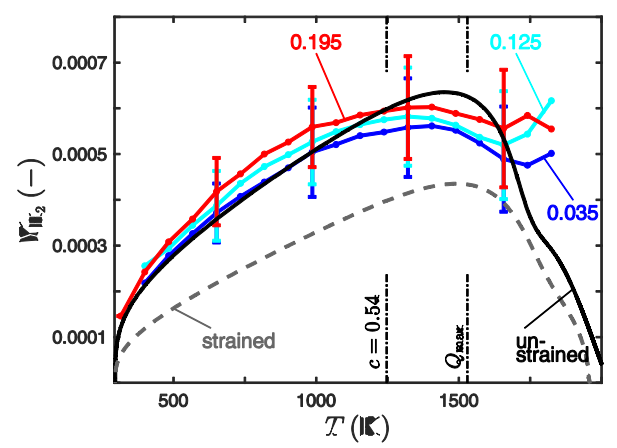

Fig. 6: $\mathrm{H}_{2}$ mass fraction in temperature space parameterized on three different levels of stratification (in $\mathrm{mm}-1$ ). Unstrained and strained $\left(1000 \mathrm{~s}^{-1}\right)$ premixed laminar flame at equivalent local equivalence ratio for comparison. Vertical lines indicate location of maximum temperature gradient $(c=0.54)$ and location of maximum heat release in temperature space for unstrained flame.

The flame structure in the preheat and reaction zone is further investigated by examining the temperature gradients (Fig. 7). Spatial filtering was applied to compute $\nabla T$ from the simulations to match the resolution of the experimental system of $60 \mu \mathrm{m}$ in direction of the flame normal. Within the preheat layer stratification enlarges the thermal gradients and these again enhance the transport towards the reactant side. This effect is visible for the largest part of the preheat-layer in the temperature range between $\sim 700 \mathrm{~K}$ and $\sim 1500 \mathrm{~K}$. The latter is approximately the temperature at the maximum heat release (indicated by a vertical line). A similar increase of $\nabla T$ by stratification was observed for the stratified Cambridge configuration by Sweeney et al. [5]. Temperature gradients for the turbulent TSFA flame in this intermediate temperature range are below the ones of strained and mostly even below the ones of unstrained laminar flames. In the thin-reaction-zone regime the preheat zone is 
perturbed and thickened by small scale turbulent eddies, which results in comparison to laminar flames to a decrease of temperature gradients $[5,9]$. This trend is visible in Fig. 7 and supported by the comparison of the turbulent and laminar temperature profiles shown in Fig. 4.

At the low temperature end (reactants) the measured temperature gradients are larger than those from the unstrained laminar flame and closely match the ones of strained laminar flames. Moreover, the impact of stratification on $\nabla T$ vanishes for low temperatures. This first finding is in contrast to Sweeney et al. [5], who report the $\nabla T$ in their turbulent flame configuration to be even smaller than the $\nabla T$ of an unstrained laminar flame. An experimental error due to a change in the flame normal derived from CRPI, as one moves away from the point at higher temperatures where it is measured, can be excluded. Such a change, which might for instance due to flame curvature effects, would result in a bias towards smaller conditional temperature gradients instead to the larger $\nabla T$ found here. Such, the comparatively large temperature gradients at small temperatures are most likely caused by the mixing characteristics of the current flow configuration. Evidently, the standard deviations of $\nabla T$ are smaller for the lower temperatures range than for the higher temperatures and indicate that the structure of the preheat layer might be stronger disrupted at higher temperatures.

$\nabla T$ is very similar for all stratification levels between the reaction zone and the products. This is in accord with the discussion of the temperatures over the flame normal coordinate (Fig. 4). The temperature gradient in the products is almost not affected by stratification after the location of the maximum heat release in temperature space has been passed. Such, small scale turbulence does not disturb the reaction layer itself to a large degree as the flow scales are still too large to penetrate into the layer. This supports again the finding that the turbulent flame is within the thin-reaction-zone regime. 


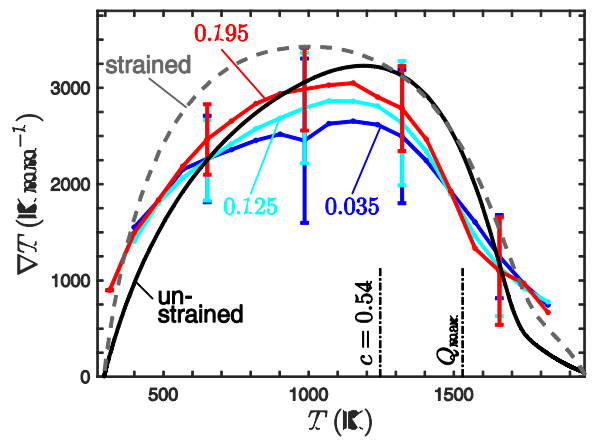

Fig. 7: Mean and standard deviation of $\nabla \mathrm{T}$ in temperature space, parameterized on three different levels of stratification (in $\left.\mathrm{mm}^{-1}\right)$. Unstrained and strained $\left(1000 \mathrm{~s}^{-1}\right)$ premixed flame at equivalent local equivalence ratio for comparison.

\section{Conclusion}

The sensitivity of lean combustion to stratification level has been investigated in a turbulent flow field for the TSFA configuration. Major species concentrations and temperature have been measured. The flame front orientation derived from CPRI was used to correct the line data and quasi 3D-temperature gradients were obtain. Data were taken at the intersection of the mean mixing layer and the flame brush to maximize the impact of stratification. These have been be conditioned on a local equivalence ratio $(\Phi=0.75 \pm 0.03)$ as well as on a local thermal progress variable $(c=0.54)$ and parameterized on the stratification level. Thereby, the effect of equivalence ratio gradients onto the flame structure were isolated. The main findings are summarized below.

- Differential diffusion caused by the flux of species such as $\mathrm{H}_{2}$ is identified for all stratification levels.

- The impact of stratification onto the flame micro-structure is not only evident in the preheat zone but also in the reaction zone as shown by the means of the local equivalence ratio. This is related to the characteristic Karlovitz and Damköhler numbers in flame TSFA.

- On the product side, higher stratification increases the temperatures only slightly and has negligible impact on $\nabla T$. Turbulence-chemistry interaction in general as well as differential diffusion enhanced by the larger gradients in stratified flames might cause that. 
- On the reactant side, stratification enhances the transport of lighter species and heat, which results in increased $\mathrm{H}_{2}$ concentrations as well as a rise in $\nabla T$.

The approach to parametrize the stratification level by applying a multiple conditioning which keeps other parameter relevant to stratified combustion constant, allows for detailed analysis. An extension of the equivalence ratio range and flame configurations at different locations in the flame regime diagram would be necessary to gain an understanding of the effects turbulence onto the flame micro-structure.

\section{Acknowledgements}

We gratefully acknowledge financial support by the Deutsche Forschungsgemeinschaft (DFG) through DR 374/13 and GE 2523/1. A. Dreizler was financially supported by the Gottfried Wilhelm Leibniz-Preis (DFG). Work at Sandia was supported by the United States Department of Energy, Office of Basic Energy Science, and Biosciences. Sandia National Laboratories is a multiprogram operated by Sandia Corporation, a Lockheed Martin Company, for the United States Department of Energy under contract DE-AC04-94-AL85000. The authors also thank Bob Harmon for his contributions to the experiments. 


\section{Figure Captions}

Figure 1:

Fig. 1: Illustration of the burner configuration and mean radial position of the center of the mixing layer $\phi_{m}=\frac{\phi_{1}+\phi_{2}}{2}$ and the center of the flame brush at $T_{\max }^{\prime}$. Location of the maximum in scalar mixing is marked by $\phi_{\max }^{\prime}$. Numbers at burner exit are respective equivalence ratios in $S_{1}, S_{2}$ and $P$.

\section{Figure 2:}

Fig 2: $\mathrm{CH}_{4}$-trajectories for $\left.\phi\right|_{c=0.54}=0.75 \pm 0.03$ over the local equivalence ratio at two different levels of stratification. Low stratification ( $\Delta$ symbols), high stratification ( $\circ$ symbols), and pure mixing (black, dash-dotted). An unstrained premixed flame at matching local equivalence ratio is included for comparison.

\section{Figure 3:}

Fig. 3: Probability density distribution of stratification conditioned for three different equivalence ratios.

\section{Figure 4:}

Fig. 4: Mean and standard deviation of temperature over flame-normal coordinate $r^{*}$, parameterized on three different levels of stratification in $\mathrm{mm}^{-1}$; low (blue), mid (turquoise), high (red). Unstrained and strained $\left(1000 \mathrm{~s}^{-1}\right)$ premixed laminar flame at equivalent local equivalence ratio for comparison.

\section{Figure 5:}

Fig. 5: Mean and standard deviation of $\phi$ in temperature space for two equivalence ratios, parameterized on three different levels of stratification (in $\left.\mathrm{mm}^{-1}\right)$. Unstrained and strained $\left(1000 \mathrm{~s}^{-1}\right)$ premixed laminar flame at equivalent local equivalence ratio for comparison. Vertical lines indicate location of maximum temperature gradient $(c=0.54)$ and location of maximum heat release in temperature space for unstrained flame. 
Figure 6:

Fig. 6: $\mathrm{H}_{2}$ mass fraction in temperature space parameterized on three different levels of stratification (in $\mathrm{mm}-1)$. Unstrained and strained $\left(1000 \mathrm{~s}^{-1}\right)$ premixed laminar flame at equivalent local equivalence ratio for comparison. Vertical lines indicate location of maximum temperature gradient $(c=0.54)$ and location of maximum heat release in temperature space for unstrained flame.

Figure 7:

Fig. 7: Mean and standard deviation of $\nabla \mathrm{T}$ in temperature space, parameterized on three different levels of stratification (in $\mathrm{mm}^{-1}$ ). Unstrained and strained $\left(1000 \mathrm{~s}^{-1}\right.$ ) premixed flame at equivalent local equivalence ratio for comparison. 


\section{References}

[1] A.R. Masri, Proc. Combust. Inst., 35 (2015) 1115-1136.

[2] G. Kuenne, F. Seffrin, F. Fuest, T. Stahler, A. Ketelheun, D. Geyer, J. Janicka, A. Dreizler, Combust. Flame, 159 (2012) 2669-2689.

[3] E.S. Richardson, V.E. Granet, A. Eyssartier, J.H. Chen, Combust. Theor. Model., 14 (2010) 775-792.

[4] P.C. Vena, B. Deschamps, H. Guo, M.R. Johnson, Combust. Flame, 162 (2015) 4231-4240.

[5] M.S. Sweeney, S. Hochgreb, M.J. Dunn, R.S. Barlow, Combust. Flame, 160 (2013) 322-334.

[6] M.M. Kamal, R.S. Barlow, S. Hochgreb, Combust. Flame, 162 (2015) 3896-3913.

[7] B. Fiorina, R. Mercier, G. Kuenne, A. Ketelheun, A. Avdić, J. Janicka, D. Geyer, A. Dreizler, E. Alenius, C. Duwig, P. Trisjono, K. Kleinheinz, S. Kang, H. Pitsch, F. Proch, F. Cavallo Marincola, A. Kempf, Combust. Flame, (2015).

[8] F. Seffrin, F. Fuest, D. Geyer, A. Dreizler, Combust. Flame, 157 (2010) 384-396.

[9] M.S. Sweeney, S. Hochgreb, M.J. Dunn, R.S. Barlow, Combust. Flame, 159 (2012) 2896-2911.

[10] G. Magnotti, R.S. Barlow, Combust. Flame, 162 (2015) 100-114.

[11] F. Fuest, R.S. Barlow, D. Geyer, F. Seffrin, A. Dreizler, Proc. Combust. Inst., 33 (2011) 815-822.

[12] V. Robin, A. Mura, M. Champion, O. Degardin, B. Renou, M. Boukhalfa, Combust. Flame, 153 (2008) 288-315.

[13] D. Goodwin, N. Malaya, H. Moffat, R. Speth, available at <https://code.google.com/p/cantera/>.

[14] G.P. Smith, D.M. Golden, M. Frenklach, B. Eiteener, M. Goldenberg, C.T. Bowman, R.K. Hanson, W.C. Gardiner, V.V. Lissianski, Z.W. Qin, available at <http://www.me.berkeley.edu/gri mech/>.

[15] Y.M. Marzouk, A.F. Ghoniem, H.N. Najm, Proc. Combust. Inst., 28 (2000) 1859-1866.

[16] P. Tamadonfar, Ö.L. Gülder, Combust. Flame, 162 (2015) 115-128. 